\title{
Precision Electroweak measurements at the Future Circular Collider
}

\author{
Mogens Dam *i \\ Niels Bohr Institute, Copenhagen University \\ E-mail: damenbi.dk
}

Electroweak precision measurements at the Future Circular Collider with electron-positron beams (FCC-ee) would provide improvements by a factor of order 25 over the present status, and constitute a broad search for the existence of new, weakly interacting particles up to very high energy scales. The $\mathrm{Z}$ mass and width, as well as the value of the electroweak mixing angle and $\mathrm{b}$ partial width, can be measured with very high precision at the $\mathrm{Z}$ pole thanks to an instantaneous luminosity five to six orders of magnitude larger than at LEP. At centre-of-mass energies around $160 \mathrm{GeV}$, corresponding to the WW production threshold, the $\mathrm{W}$ mass can be determined very precisely with high-statistics cross section measurements at several energy points. This exceptional performance can be achieved thanks to continuous beam energy calibration by resonant depolarisation of the beams. Considerable improvements of the strong coupling constant determination down to a precision of $\Delta \alpha_{\mathrm{s}}\left(m_{\mathrm{Z}}\right) \simeq \pm 0.0001$ will be possible via the measurements of the hadronic widths of the $\mathrm{Z}$ and $\mathrm{W}$ bosons. Similarly, a very precise determination of the top-quark mass can be provided by an energy scan around the $350 \mathrm{GeV} t \bar{t}$ production threshold.

The European Physical Society Conference on High Energy Physics

22-29 July 2015

Vienna, Austria

* Speaker.

${ }^{\dagger}$ On behalf of the FCC Design Study Group. 


\section{Introduction}

The discovery in $2012[1,2]$ of the Higgs boson at the relatively low mass of $125 \mathrm{GeV} / \mathrm{c}^{2}$ has revived the interest in circular electron-positron colliders that can serve as Higgs factories. The design study [3] of the Future Circular Collider (FCC) in a 100-km tunnel in the Geneva area was initiated by CERN in early 2014. Whereas the ultimate goal of the FCC programme is a $100 \mathrm{TeV}$ proton-proton collider, a high-luminosity $\mathrm{e}^{+} \mathrm{e}^{-}$collider, FCC-ee, able to address centre-of-mass energies ranging from below the $\mathrm{Z}$ pole to the t⿱亠幺 production threshold and above is a possible first step. The use of techniques inspired from b-factories, very strong focussing combined with full-energy top-up injection into separate $\mathrm{e}^{+}$and $\mathrm{e}^{-}$storage rings, allows the FCC-ee to achieve very high luminosities over the targeted energy range. Compared to the equivalent linear collider projects, the advantage of the FCC-ee is the higher luminosity at the targeted energies and the possibility to instrument several interaction points. Assuming four interaction points, the luminosity advantage is about tenfold for the Higgs-strahlung process $\mathrm{e}^{+} \mathrm{e}^{-} \rightarrow \mathrm{ZH}$ at $E_{\mathrm{CM}}=240 \mathrm{GeV}$ increasing steeply at lower energies [4]. In summary, the FCC-ee programme aims at collecting $10^{12}$ (possibly $10^{13}$ with the crab-waist scheme [5]) $\mathrm{Z}$ decays in an energy scan around the $\mathrm{Z}$ mass, $2 \times 10^{8} \mathrm{~W}$ pairs at and above production threshold, $10^{6}$ Higgs decays at $240 \mathrm{GeV}$, and $10^{6} \mathrm{t} \overline{\mathrm{t}}$ pairs at and just above threshold, see Refs. [6, 7, 8] for further details.

The FCC-ee offers a very broad physics programme [6, 7]. In this report, I discuss the prospects for precision electroweak measurements at the $\mathrm{Z}$ pole and at the WW production threshold. Top quark properties, mass and couplings, can be determined with very high precision at the $\bar{t} \bar{t}$ production threshold. Here, only the mass measurement will be mentioned; a dedicated discussion of top-quark couplings can be found in [9] and elsewhere in these proceedings [8]. Also in these proceedings can be found discussions of the prospects for the FCC-ee as a Higgs factory [10] and for high precision flavour physics measurements [11].

\section{Electroweak precision measurements in the Higgs era}

Electroweak loops have the remarkable property of being sensitive to the existence of weaklycoupled particles, even it these cannot be directly produced or observed in current experiments. Historically, electroweak precision measurements have been instrumental in predicting and determining free parameters of the Standard Model. Now, with the Higgs boson discovered, all SM particles are known, and, within the SM, there are no free knobs left to turn. Hence, precision electroweak measurements now have a new target: If inconsistencies appear between measurements, this will point directly to Beyond Standard Model Physics.

\section{Physics at the $\mathrm{Z}$ pole}

One of the cornerstones of electroweak precision measurements is the determination of the mass and width of the $\mathrm{Z}$ boson. These were determined at LEP via the line-shape scan to precisions of $2 \mathrm{MeV}$ each. Key to this measurement is a precise knowledge of the centre-of-mass energy, which by far dominates the systematic uncertainty on the Z-mass. At LEP, a relative precision of $2 \times 10^{-5}$ was reached [12] with the technique of resonant depolarisation [13]. A detailed discussion of this technique for FCC-ee can be found elsewhere in these proceedings [4]. 
It was found at LEP, that the intrinsic precision of each individual beam energy measurement was $100 \mathrm{keV}$ or better. Since, however, this measurement was only performed in dedicated polarisation runs, the final uncertainty was more than tenfold larger because the measurements had to be transported to the collision runs. At FCC-ee, it will be possible to exploit the full precision of the technique by keeping a few non-colliding bunches and applying regular resonant spin depolarisation on those. This would lead to uncertainties of $100 \mathrm{keV}$ on both the $\mathrm{Z}$ mass and width.

The five to six order of magnitude increased statistics compared to LEP will allow also for much improved measurements of other Z-pole observables such as $\mathrm{Z}$ partial widths and numerous asymmetries sensitive to the weak mixing angle. The benefit of the increased statistics can, in particular, be reaped for the measurement of ratios, where systematic effects tend to cancel. Important examples are the ratio between the hadronic and leptonic decay widths, $R_{\ell}$, and the ratio between the b-quark and the total hadronic widths, $R_{\mathrm{b}}$. For these two ratios, respectively, relative precision of $5 \times 10^{-5}$ and of $3 \times 10^{-4}$ seem achievable. This bears importance for the determination of the strong coupling constant, as detailed below.

At LEP, the determination of the weak mixing angle, $\sin ^{2} \theta_{\mathrm{W}}^{\text {eff }}$, was based on a variety of measurements such as the leptonic and hadronic forward-backward asymmetries and the $\tau$ polarisation in $\mathrm{Z} \rightarrow \tau \tau$ decays. The single most precise measurement, however, came from the SLD experiment from the inclusive left-right beam-polarisation asymmetry, $A_{\mathrm{LR}}$. Studies are ongoing to understand whether FCC-ee would be able to operate with longitudinally polarised beams, and whether, in this case, one would be still able to maintain the required precision on the beam energy calibration. Even without longitudinally polarised beams, the increased statistics of FCC-ee will lead to a sizeable improvement compared to the LEP measurements, which were largely statistics limited. Detailed studies of the various asymmetry measurements are under way. An first study of the forward-backward asymmetry for muon pairs, $A_{\mathrm{FB}}^{\mu \mu}$, indicates that an improvement over LEP by a factor 50 is within reach. This matches well, the $100 \mathrm{keV}$ uncertainty on the centre-of-mass energy, which enters into the extraction of $\sin ^{2} \theta_{\mathrm{W}}^{\mathrm{eff}}$ from the $A_{\mathrm{FB}}^{\mu \mu}$ measurement.

The very large statistics accumulated at FCC-ee should allow a new range of searches for very rare phenomena and tests of conservation laws that remain to be investigated. An example, is the search for sterile right-handed partners of neutrinos in $\mathrm{Z}$ decays $[14,7]$.

\section{Physics at the WW production threshold}

With $O\left(10^{8}\right) \mathrm{W}$ pairs collected at the production threshold and above, FCC-ee will be able to provide very precise measurements of W-boson properties, such as mass, width, and branching fractions.

For the determination of the $\mathrm{W}$ mass and width, operation at a few energy points within one or two $\mathrm{GeV}$ from production threshold is particularly interesting, since, in this narrow region, the WW cross section is maximally sensitive to the values of the $\mathrm{W}$ mass and width. Again, to perform precise measurements, an accurate calibration of the centre-of-mass energy is necessary. Transverse beam polarisation builds up naturally in a storage ring by the Sokolov-Ternov effect [15]. To maintain polarisation at a useful level, particles have to avoid depolarising resonances spaced by $440.7 \mathrm{MeV}$ in beam energy. Since the beam-energy spread increases with beam energy this effectively sets an upper limit on the energy at which a useful polarisation level can be maintained. At 
LEP the maximum beam energy where polarisation was observed was $60.6 \mathrm{GeV}$. Extrapolating this to FCC-ee, taking into account that the beam energy spread scales approximately as $\sigma_{E} \propto E^{2} / \sqrt{\rho}$, where $\rho$ is the bending radius, indicates that beam polarisation should be available at the WW threshold, with the potential of achieving very precise measurements of the $\mathrm{W}$ mass and width. A precision of $300 \mathrm{keV}$ on the $\mathrm{W}$ mass seems within reach.

\section{The top-quark mass}

The FCC-ee will enable precise top-quark studies as well, with over $10^{6} \mathrm{t} \overline{\mathrm{t}}$ pairs produced at and above production threshold in a very clean experimental environment. An important achievement will be a sizeable improvement in the measurement of the top-quark mass, which is currently determined at hadron colliders with an experimental precision of about $0.5 \%$, dominated by systematic effects. At hadron colliders, the mass is determined from the invariant mass of the decay products. Because of colour reconnection between final state hadrons these cannot unambiguously be related to a single top quark, and hence the method is affected by an inherent uncertainty of the order of $\Lambda_{\mathrm{QCD}} \sim 500 \mathrm{MeV}$ in the theoretical interpretation of the experimental measurement.

From precise measurements of the t⿱t production cross section in a narrow scan region around threshold, the top-quark mass can be determined with a statistical precision of the order of $10 \mathrm{MeV}$. The measurement benefits from the precisely defined centre-of-mass energy, which, unlike at linear colliders, is not affected by strong beamstrahlung effects. The threshold behaviour of the cross section has a sizable dependence on the strong coupling constant, and hence the measurement will profit from the very precise independent determination of $\alpha_{\mathrm{s}}$ at the $\mathrm{Z}$ and WW running, see below. The threshold scan method is characterised by well-controlled theory uncertainties, likely making it the ultimate measurement of the top-quark mass.

\section{The strong coupling constant}

At LEP, a precise measurement of $\alpha_{\mathrm{s}}\left(m_{\mathrm{Z}}^{2}\right)$ was derived from the $\mathrm{Z}$ decay width ratio $R_{\ell}=\Gamma_{\text {had }} / \Gamma_{\ell}$. Reinterpreting this measurement in the light of $i$ ) new $\mathrm{N}^{3} \mathrm{LO}$ calculations, ii) the improved knowledge of $m_{\text {top }}$, and iii) the knowledge of $m_{\mathrm{Higgs}}$, the uncertainty can be now expressed as $\delta\left(\alpha_{\mathrm{s}}\left(m_{\mathrm{Z}}^{2}\right)\right)_{\mathrm{LEP}}= \pm 0.0038$ (exp) \pm 0.0002 (other), and is thus dominated by experimental effects. At FCC-ee, with the much improved experimental precision on $R_{\ell}$, a precision on $\alpha_{\mathrm{s}}\left(m_{\mathrm{Z}}^{2}\right)$ of 0.00015 would seem achievable.

In a similar way, $\alpha_{\mathrm{s}}\left(m_{\mathrm{W}}^{2}\right)$ can be derived from a precise measurement of the hadronic branching fraction of the W boson, $B_{\text {had }}=\left(\Gamma_{\text {had }} / \Gamma_{\text {tot }}\right)_{\mathrm{W}}$. At LEP, with $4 \times 10^{4} \mathrm{~W}$-pair events, this quantity was measured with a relative precision of $0.4 \%$, not precise enough for an interesting constraint on $\alpha_{\mathrm{s}}$. With a factor $O\left(10^{4}\right)$ more data at FCC-ee, an improvement of up to two orders of magnitude on $B_{\text {had }}$ can be foreseen, resulting in a absolute uncertainty on $\alpha_{\mathrm{s}}\left(m_{\mathrm{W}}^{2}\right)$ of \pm 0.00015 , thus matching the uncertainty derived from $\mathrm{Z}$ decays. Combining the $\mathrm{Z}$ and $\mathrm{W}$ measurements, a precision of 0.0001 seems within reach for $\alpha_{\mathrm{s}}\left(m_{\mathrm{Z}}^{2}\right)$. 


\section{The $Z$ invisible width and the number of neutrinos}

At LEP, the measurement of the $\mathrm{Z}$ peak hadronic cross-section led to the determination of the number of active neutrino species, $N_{v}=2.984 \pm 0.008$, which can be observed to be two standard deviations below the SM value of 3 . This measurement is of great interest as it constitutes a direct test of the unitarity of the PMNS matrix - or of the existence of sterile neutrinos, as pointed out in Ref. [16]. The experimental conditions at FCC-ee will be adequate to improve the experimental uncertainty considerably, but, to make the measurement worthwhile, a commensurate effort would have to be invested in the theoretical calculation of the small-angle Bhabha-scattering cross section used for the normalisation. Indeed, the current measurement is limited by the theoretical understanding of this process. A desirable goal would be to reduce the uncertainty on $N_{v}$ down to 0.001 , but it is not clear that this can be achieved from the $\mathrm{Z}$ peak measurement.

Potentially more promising is the use of radiative return processes at centre-of-mass energies above the $\mathrm{Z}$ pole, i.e. the process $\mathrm{e}^{+} \mathrm{e}^{-} \rightarrow \mathrm{Z} \gamma$, leading to a very clean photon-tagged sample of on-shell $\mathrm{Z}$ bosons, with which $\mathrm{Z}$ properties can be measured. From the WW threshold scan alone, 10 million $Z \gamma$ events with $Z \rightarrow v \bar{v}$ and a photon inside the detector acceptance will be produced per experiment. Adding the statistics from the 240 and $350 \mathrm{GeV}$ running, the invisible width can be measured with a statistical precision corresponding to 0.001 on $N_{v}$. Recently, it has been suggested to search for the direct $s$-channel production of Higgs bosons in a dedicated high-luminosity run at $E_{\mathrm{CM}}=125 \mathrm{GeV}$, see [10]. Such a run would be ideal for the the neutrino counting exercise and could reduce the statistical uncertainty by about a factor of two relative the runs at higher energies.

\section{Potential of a direct measurement of $\alpha_{\mathrm{QED}}$ at the Z-mass scale}

The exceptional experimental precision in the $\mathrm{Z}, \mathrm{W}$, and top-quark measurements will require a matching precision on the determination of the electromagnetic coupling constant at the Z-mass scale relevant for electroweak interactions, $\alpha_{\mathrm{QED}}\left(m_{\mathrm{Z}}^{2}\right)$, in order to exploit the full potential of the FCC-ee to unravel signs of new physics. The current determination of $\alpha_{\mathrm{QED}}\left(m_{\mathrm{Z}}^{2}\right)$ is based on the measurement of the fine structure constant at zero momentum transfer, $\alpha_{\mathrm{QED}}(0)$, followed by an extrapolation to the Z-mass scale. The uncertainty on this extrapolation, involving photon selfenergy diagrams, is dominated by the hadronic part, which is obtained by a dispersion relation over measured low energy hadronic cross sections in $\mathrm{e}^{+} \mathrm{e}^{-}$annihilations. Current calculations result in a relative uncertainty on $\alpha_{\mathrm{QED}}\left(m_{\mathrm{Z}}^{2}\right)$ of $1.1 \times 10^{-4}$ [17]. An improvement by at least a factor of five is called for to match the FCC-ee experimental precision.

It has been recently demonstrated [18] that the formidable statistics of the FCC-ee could allow a direct measurement of $\alpha_{\mathrm{QED}}$ close to the Z-mass scale, eliminating the need for extrapolation from zero momentum transfer. The study exploits the $\mathrm{e}^{+} \mathrm{e}^{-} \rightarrow \mathrm{Z} / \gamma^{*} \rightarrow \ell^{+} \ell^{-}$process. At the peak of the $\mathrm{Z}$ resonance, this is completely dominated by $\mathrm{Z}$ exchange and has no sensitivity to $\alpha_{\mathrm{QED}}$. Away from the peak, however, $\gamma^{*}$ exchange gradually takes over, and the sensitivity to $\alpha_{\mathrm{QED}}$ increases. Two observables have been investigated: The total production cross section, $\sigma_{\mu \mu}=\sigma\left(\mathrm{e}^{+} \mathrm{e}^{-} \rightarrow \mu^{+} \mu^{-}\right)$and the forward-backward asymmetry, $A_{\mathrm{FB}}^{\ell \ell}$, with $\ell=\mu, \tau$. Figure 1 summaries the study in terms of the achievable statistical precision on $\alpha_{\mathrm{QED}}$ after one year of running at any energy point in a wide region around the $\mathrm{Z}$ pole. Whereas the statistical uncertainty 


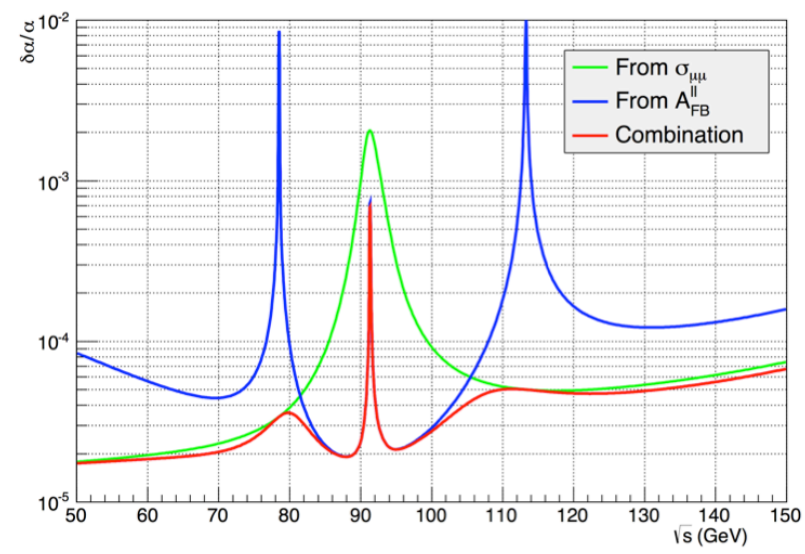

Figure 1: Relative statistical uncertainty on $\alpha_{\mathrm{QED}}$ versus centre-of-mass energy. One year of running at any energy point is assumed.

from the $\sigma_{\mu \mu}$ measurement continues to decrease away from the $\mathrm{Z}$ pole, the uncertainty from the $A_{\mathrm{FB}}^{\ell \ell}$ measurement shows a more interesting pattern. In fact, the best relative precision on $\alpha_{\mathrm{QED}}$ of $2 \times 10^{-5}$ appears at centre-of-mass energies of 88 and $95 \mathrm{GeV}$, i.e. only $\pm 3.5 \mathrm{GeV}$ away from the $\mathrm{Z}$ peak, energies which could be easily accommodated in the course of the Z-resonance scan. It is important to note that the large parametric uncertainty from $\sin ^{2} \theta_{\mathrm{W}}^{\text {eff }}$, which influences the measurement, goes in opposite directions at the 88 and $95 \mathrm{GeV}$ energy points. Thus, it largely cancels if one averages over these two points. Experimental uncertainties, which are expected to be small, are to be evaluated in detail.

\section{Summary}

The proposed FCC-ee, a large circular $\mathrm{e}^{+} \mathrm{e}^{-}$collider delivering very high luminosities at centre-ofmass energies from 90 to $350 \mathrm{GeV}$ and above, will allow measurements of electroweak observables at an unrivalled level of precision. Potentially it could provide measurements of the $\mathrm{Z}$ mass and width to a precision of $100 \mathrm{keV}$ each, the $\mathrm{W}$ mass to $300 \mathrm{keV}$, and the top-quark mass to $10 \mathrm{MeV}$. These and other selected electroweak precision measurements at the FCC-ee are summarised in Table 1, where the quoted systematic uncertainties are indicative and will be revisited in the course of the ongoing design study. If these goals are achieved, the contour line in the $\left(m_{\mathrm{top}}, m_{\mathrm{W}}\right)$-plane could evolve from today to FCC-ee as indicated in Figure 2, where both the results of the direct mass measurements and the indirect constraints from the precision $\mathrm{Z}$ pole measurements are indicated. With all of the Standard Model parameters precisely known, the prediction of a number of observables sensitive to electroweak radiative predictions become absolute, and any deviation between measurements would be a demonstration of the existence of new, weakly interacting particles. With the dramatic increase in precision, sensitivity to new physics up to energy scales of the order of $30 \mathrm{TeV}$ can be envisioned as shown in the recent paper [19]. 


\begin{tabular}{|c|c|c|c|c|c|}
\hline Observable & Measurement & Current precision & FCC-ee stat. & Possible syst. & Challenge \\
\hline \hline$M_{\mathrm{Z}}[\mathrm{MeV}]$ & Lineshape & $91187.5 \pm 2.1$ & 0.005 & $<0.1$ & QED corr. \\
\hline$\Gamma_{\mathrm{Z}}[\mathrm{MeV}]$ & Lineshape & $2495.2 \pm 2.3$ & 0.008 & $<0.1$ & QED corr. \\
\hline$R_{\ell}$ & Peak & $20.767 \pm 0.025$ & 0.0001 & $<0.001$ & Statistics \\
\hline$R_{\mathrm{b}}$ & Peak & $0.21629 \pm 0.00066$ & 0.000003 & $<0.00006$ & $g \rightarrow \mathrm{b} \overline{\mathrm{b}}$ \\
\hline$N_{v}$ & Peak & $2.984 \pm 0.008$ & 0.00004 & 0.004 & Lumi meast. \\
\hline$A_{\mathrm{FB}}^{\mu \mu}$ & Peak & $0.0171 \pm 0.0010$ & 0.000004 & $<0.00001$ & $E_{\text {beam }}$ meast. \\
\hline$\alpha_{\mathrm{s}}\left(M_{\mathrm{Z}}\right)$ & $R_{\ell}$ & $0.1190 \pm 0.0025$ & 0.000001 & 0.00015 & New Physics \\
\hline$M_{\mathrm{W}}[\mathrm{MeV}]$ & Threshold scan & $80385 \pm 15$ & 0.3 & $<1$ & QED corr. \\
\hline$N_{v}$ & $\mathrm{e}^{+} \mathrm{e}^{-} \rightarrow \gamma \mathrm{Z}($ inv. $)$ & $2.92 \pm 0.05$ & 0.0008 & $<0.001$ & $?$ \\
\hline$\alpha_{\mathrm{s}}\left(M_{\mathrm{W}}\right)$ & $B_{\text {had }}=\left(\Gamma_{\text {had }} / \Gamma_{\text {tot }}\right)_{\mathrm{W}}$ & $B_{\text {had }}=67.41 \pm 0.27$ & 0.00018 & 0.00015 & CKM Matrix \\
\hline$m_{\mathrm{top}}[\mathrm{MeV}]$ & Threshold scan & $173200 \pm 900$ & 10 & 10 & QCD \\
\hline
\end{tabular}

Table 1: Selected set of precision measurements at FCC-ee. The systematic uncertainties are indicative and will be revisited in the course of the design study.

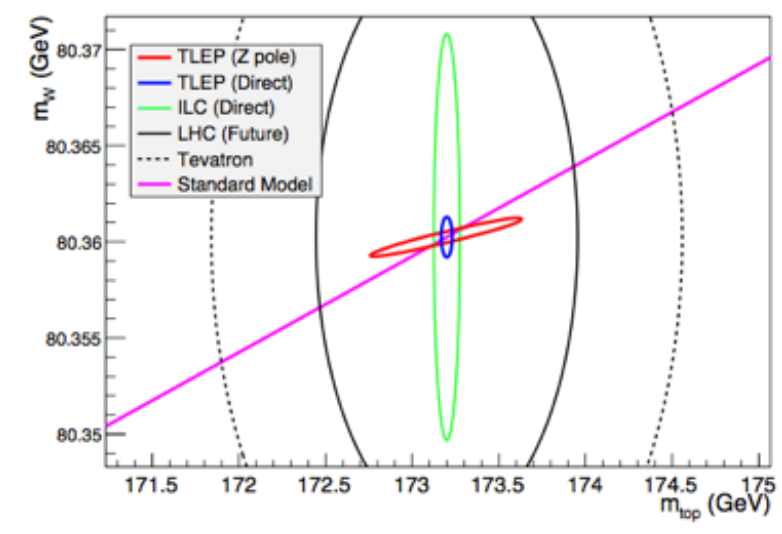

Figure 2: The $68 \%$ c.l. contour in the $\left(m_{\mathrm{W}}, m_{\mathrm{top}}\right)$-plane as expected for the FCC-ee (indicated as TLEP in the figure) and other accelerators. The blue line indicates the expected contour from direct $\mathrm{W}$ and top-quark mass measurements, while the red line gives the expected precision from a fit to the Z-pole observables.

\section{Acknowledgements}

I would like to thank Alain Blondel, Patrick Janot, and Roberto Tenchini for their help in preparing the talk.

\section{References}

[1] ATLAS Collaboration, Observation of a new particle in the search for the Standard Model Higgs boson with the ATLAS detector at the LHC, Phys.Lett. B716 (2012) 1-29.

arXiv:1207.7214, doi:10.1016/j.physletb.2012.08.020.

[2] CMS Collaboration, Observation of a new boson at a mass of $125 \mathrm{GeV}$ with the CMS experiment at the LHC, Phys.Lett. B716 (2012) 30-61.

arXiv:1207.7235, doi:10.1016/j.physletb.2012.08.021. 
[3] The FCC design study, http:// cern. ch/fcC

[4] M. Koratzinos, FCC-ee energy and luminosity, poster presented at EPS-HEP2015, Vienna, 22-29 July 2015, these proceedings.

[5] A. Bogomyagkov, E. Levichev, D. Shatilov, Beam-beam effects investigation and parameters optimization for a circular $e^{+} e^{-}$collider at very high energies, Phys.Rev. ST Accel. Beams 17 (2014) 041004. doi:10.1103/PhysRevSTAB.17.041004.

[6] M. Bicer, et al., First Look at the Physics Case of TLEP, JHEP 1401 (2014) 164. arXiv:1308.6176. doi:10.1007/JHEP01(2014)164.

[7] A. Blondel, Physics at the FCC-ee, poster presented at EPS-HEP2015, Vienna, 22-29 July 2015, these proceedings.

[8] P. Janot, Top couplings at the FCC, talk presented at EPS-HEP2015, Vienna, 22-29 July 2015, these proceedings.

[9] P. Janot, Top-quark electroweak couplings at the FCC-ee, JHEP 04 (2015) 182.

[10] M. Klute, Higgs phyiscs at the FCC, talk presented at EPS-HEP2015, Vienna, 22-29 July 2015, these proceedings.

[11] S. Monteil, Flavours at the FCC-ee, talk presented at EPS-HEP2015, Vienna, 22-29 July 2015, these proceedings.

[12] The ALEPH, DELPHI, L3, OPAL, SLD Collaborations, the LEP Electroweak Working Group, the SLD Electroweak and Heavy Flavour Groups, Precision Electroweak Measurements on the Z Resonance, Phys. Rep. 427 (2006) 257-454. arXiv: hep-ex/0509008, doi:10.1016/j.physrep.2005.12.006

[13] L. Arnaudon et al., Accurate determination of the LEP beam energy with resonant depolarization, Z.Phys. C66 (1995) 45-62. doi : 10 . 1007 /BF 01496579.

[14] A. Blondel, E. Graverini, N. Serra, M. Shaposhnikon, Search for Heavy Right Handed Neutrinos at the FCC-ee, arXiv: 1411.5230.

[15] A. A. Sokolov, I. M. Ternov, On Polarization and spin effects in the theory of synchrotron radiation, Sov. Phys. Dokl. 8, 1203 (1964).

[16] C. Jarlskog, Neutrino counting at the Z-peak and right-handed neutrinos, Phys. Lett. B241, 579-583 (1990), http://dx.doi.org./10.1016/0370-2693(90) 91873-A.

[17] J. Erler and A. Freitas, Electroweak Midel and Constraints on New Physics, in K. A. Olive et al. (PDG), Chin. Phys. C38, 090001 (2014), http: / / pdg . lbl . gov.

[18] P. Janot, Determination of $\alpha_{\mathrm{QED}}\left(M_{\mathrm{Z}}\right)$ at FCC-ee, presentation at FCC-ee Physics Meeting, 29 June 2015, https://indico.cern.ch/event/401698/

[19] J. Ellis and T. You, Sensitivities of Prospective Future $e^{+} e^{-}$Colliders to Decoupled New Physics, arXiv:1510.04561 